\section{Designing well-connected marine reserves for climate-change resilience with low socio-economic costs.}

The theory behind networks of marine reserves is that they allow protected pathways for species to grow and maintain populations through their lifecycle - from where individuals begin their lives (typically as planktonic larvae) to where they disperse and live later as adults. Individual reserves - patches of protected habitat - are linked together, often by oceanic currents, with organisms moving between the reserves. Connections between marine reserves in a common area can be explored with graph theory: visualizing ecological networks much like a concept map.

The ocean is warming due to climate change. Warming water has effects on larval physiology of at least some species, including shortening the larval development period, and thus shortening larval dispersal distance. These changes could theoretically compromise the connectivity and performance of marine reserve networks, thus requiring adjusting their design to account for ocean warming.

The authors used the Midriff Islands Region in the Gulf of California, Mexico as a case study to examine the effects of ocean warming on the ability of planktonic larvae to spread amongst reserves. The Midriff Islands Region features a counter-clockwise gyre in the spring and summer months, which reverses in the fall and winter. As such, larval dispersal changes depending on the time of year and where a reserve is located in relation to the center of the gyre.

Using the tools MaxEnt and Marxan, ecological features were mapped and analyzed under current and future ocean conditions. First, a network of marine reserves with the goal of uniform representation of features was modeled. Next, a second reserve network was modeled based on the first but with a secondary goal of maximizing larval dispersal under current ocean conditions. Finally, a third marine reserve network was modeled, this time optimized for connectivity based on future (ocean-warmed) conditions.

Rocky-reef ecosystems, mangroves, Sargassum forests, seagrass meadows, rhodolith beds, and coastal wetlands were all part of the analysis. Spawning aggregations for 10 fish and invertebrate species were analyzed, as were distribution patterns of 98 fish and 87 invertebrate species. Specific species distributions, however, were not modeled under future ocean conditions due to lack of available data. Socioeconomic costs were taken into consideration with the closure of existing fishing areas. Using Marxan, a maximum opportunity cost (i.e. the loss of potential catch at market value) was set at $5 \%$.

Results show that more, denser marine reserves can facilitate larval transport under climate-change scenarios with minimal socioeconomic costs. To maximize network effects, marine reserves best suited for future ocean conditions needed to be closer together (22-42\% more than the model that did not take connectivity into account). More and larger reserves were also required (approximately $25 \%$ more reserves). Marine reserves can, and should, take changing ocean conditions into account to ensure they are able to offer refuge for species. As this analysis shows, more numerous and larger marine reserves situated closer together can facilitate larval transport with minimal socioeconomic costs.
This is a summary of: Designing connected marine reserves in the face of global warming

Accessible at:

https://marxiv.org/r6tme

Authors:

Jorge Álvarez-Romero,

Adrián Munguía-Vega, Maria Beger, et al.

Added to MarXiv:

February 2018

Published: Global Change Biology, 2018

Suggested Citation: Designing well-connected marine reserves for climate-change resilience with low socio-economic costs. OCTO (2018). DOI: 10.17605/OSF.IO/EU56S

See more MarXiv summaries at https://www.marxivinfo.org/ summaries

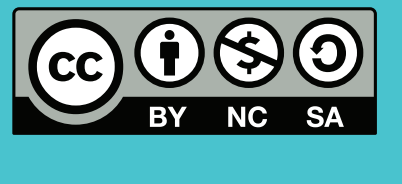

MarXiv is an ОСТO Initiative

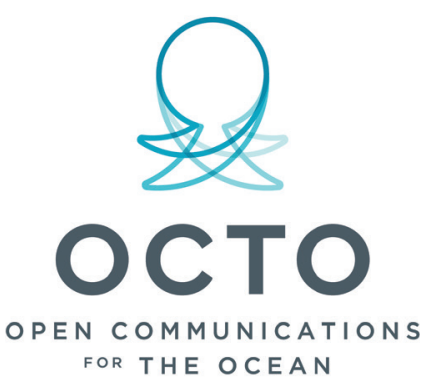

